\title{
Identification, discrimination, and match-to-sample as instances of comparative judgment
}

\author{
JEROME SMITH and HERBERT KALFMAN \\ University of Connecticut, Storrs, Connecticut 06268
}

\begin{abstract}
Identification, discrimination, and match-to-sample paradigms are discussed in the context of a comparative judgment between an external stimulus and a memorial representation. An experiment comparing the paradigms at different levels of stimulus dimensionality is described. The absence of clear-cut interactions of the paradigms with dimensionality differences is offered as support for the contention that the three seemingly diverse procedures may be considered as part of a comparative judgment continuum, with performance differences reflecting increased memorial demands.
\end{abstract}

The experiment reported here was initiated by certain procedural problems which arose in the study of the identification process in retarded and normal children (Smith \& Kaufman, 1972; Smith, Kaufman, Frost, \& Dutch, 1975). These problems stemmed from the fact that the conventional identification procedure relies heavily on verbal response and that small children, particularly those diagnosed as retarded are often not verbally competent. In the identification procedure, stimuli in a set (usually varying along one or more dimensions) are presented one at a time and the subject is asked to distinguish each stimulus with a unique response, usually a verbal one. In order to eliminate problems of verbal responding with children, we used a matching task (match-to-sample) which required a pointing response. A stimulus was introduced for identification and, following an experimentally manipulated delay period, a display matrix consisting of all the stimuli in the set was presented. The child was required to point to the one he or she had previously seen.

Our rationale for the use of the match-to-sample procedure was that while it was procedurally different from the identification (absolute judgment) task, it enabled us to study stimulus distinctiveness just as well.

The general idea of stimulus distinctiveness is that of identifiability; a stimulus is distinctive to the degree that it can be identified as that particular stimulus and not confused with other "similar" stimuli. Distinctiveness in this sense is a property of stimuli in a set, i.e., the identifiability of $A_{i}$ depends on what $A_{j}$ are possible. For this reason, "distinctiveness" is usually measured as a parameter

This research was supported by Office of Education Grant OEG-1-70-0101. The authors are indebted to Susan Dutch, Raymond Frost, and Patrick McGuire for aid in apparatus construction and data collection. Reprint requests may be sent to either author at the Department of Psychology, University of Connecticut, Storrs, Connecticut 06268. of a set of stimuli. For a number of reasons, the informational measure, $T$, the (average) bits/stimulus in a specified set, is widely taken as an index, or an operational definition, of the distinctiveness of the set, where the data, in the form of a "confusion matrix," are generated by an identification response. We felt that the match-to-sample response involved the same basic identification process as conventional verbal labeling procedures and therefore took the $T$ measure defined on the confusion matrix obtained under match-to-sample conditions as an index of distinctiveness entirely comparable to that obtained with verbal labeling. We reasoned that either paradigm would reflect the effects upon distinctiveness of manipulations such as the nature and number of dimensions varying among stimuli in a set.

George Miller (1962, pp. 147-159) points out that identification can be conceived of as a matching process in which the "sensory input is matched, simultaneously and successively, against many remembered images or schemata." Such a matching or comparison model leads naturally to questions about the nature of the input, the nature of the memorial store or schemata, and the time interval between the storage of a schema and its utilization. When these questions are posed about particular procedures, e.g., identification and match-to-sample, differences become clearer and theoretical questions arise about the nature of the process or processes underlying the procedures.

Identification and match-to-sample differ considerably when viewed as comparison of an input with some sort of memorial representation. In the identification paradigm, the input is a single stimulus, which must be compared to the schema representing the entire set of stimuli presented at the start of the experimental session and stored throughout that session. In the match-to-sample procedure, the input may be considered the entire array of stimuli which is compared to the single sample stimulus in memory which was stored just prior to the presentation of that input array. On the basis of a consideration 
in terms of comparative judgment, it would not be surprising to find match-to-sample performance exceeding that of identification, since the memory demands are smaller. This should be increasingly true as other task memory demands grow (e.g., stimulus set size increases). However, whether or not the two paradigms represent different psychological processes is a different question. Differences in performance under a single set of conditions do not necessarily mean different processes.

What constitutes adequate grounds for either distinguishing discrete psychological processes as underlying different paradigms or postulating a single process as underlying both is not easily specified. One means is to define a set of fairly specific theoretical statements and then to demonstrate how performance under the two paradigms may be dealt with under the same set of assumptions. Another means is to compare two paradigms across different types of experimental variations. If paradigm difference interacts strongly and meaningfully with different experimental variations, then grounds for inferring different processes are strong. If only simple magnitude (noninteractive) differences are found, and, further, these are eliminated in some situations, then the grounds for inferring different processes are weaker.

A preliminary study was undertaken in which an identification procedure was compared with a matchto-sample procedure with number of dimensions of stimulus variation and delay of response as experimental manipulations. No interaction of number of dimensions and type of procedure was found. As might have been anticipated, delay effects which were found in the match-to-sample condition of our earlier studies were found once again, but did not appear in the identification situation. Since the identification response is made by the subject upon stimulus presentation, requiring a delay before vocalization or recording of that response should not and did not produce any difference.

In the present study, identification and match-tosample are again compared, but this time two additional paradigms are examined as well, the four falling along a conceptual continuum within the framework of comparative judgment. At one end of the continuum lies the identification paradigm previously described. Here a small single-item sensory input is compared to a large memorial store, which has been in memory at least since the beginning of the experimental session and has been refreshed or updated only by the single item presentations of previous identification trials. At the other end of the continuum lies a paradigm in which the sensory input is compared, not to a memorial store which has been under the influence of temporal and context factors, but to another sensory input which remains constant from trial to trial and represents an external representation of the memory schema. This is a response matrix which is now presented simultaneously with the stimulus for identification. The subject need merely select which of the members of the matrix matches the stimulus. This paradigm is similar to the match-to-sample case described earlier, but differs in the crucial respect that the item to be matched is not stored in memory but is available externally along with the matrix. That this procedural difference is a conceptually large one is attested to by the fact that the paradigm of which we have been speaking has conventionally been called discrimination, and has long been considered quite distinct from the identification paradigm. Furthermore, it has not been considered within the match-to-sample context at all.

With identification at one end of the comparative judgment continuum, discrimination at the other, and match-to-sample somewhere in between, the study includes another point closer to the identification end and falling between it and match-to-sample. In this paradigm, an identification response is required but the matrix is presented on each trial prior to the presentation of the single stimulus. Here the small sensory input must be compared to a large memory schema, as is the case in identification, but the schema is, in this final paradigm, refreshed at the beginning of each trial. In terms of memory demands of each type of comparative judgment, then, it would appear that identification requires the greatest and discrimination the least, with match-tosample falling closer to discrimination and the matrix-before condition falling closer to identification.

Consistent with our concern for the interaction of comparative judgment paradigms with conditions of dimensional variation, we investigated three stimulus dimensions which we varied singly and in combination.

\section{METHOD}

\section{Subjects}

The subjects were eight undergraduates at the University of Connecticut who were paid $\$ 2 / h$ for their participation. All subjects served in all experimental conditions.

\section{Apparatus and Stimuli}

The basic apparatus was a large board, $30 \times 30 \mathrm{in} .(.76 \times$ $.76 \mathrm{~m}$ ), mounted vertically on an easel and placed at approximately eye level at a distance of $3 \mathrm{ft}$ from the seated subject. A set of 16 stimulus cards, $3 \times 5$ in. $(.07 \times .127 \mathrm{~m})$, were inserted in clips forming a circular pattern (the "matrix") of all the possible stimuli of a given experimental condition. A holder for the test stimulus was constructed in the center of the circular pattern. The test stimulus could be inserted from the rear of the display board.

A mask cut from Masonite could be moved from right to left or from left to right in front of the display board. The mask was 
patterned so that one position revealed the central test stimulus while the surrounding matrix was covered. Movement of the mask to the right would display the matrix and cover the test stimulus. Further movement would uncover the entire board, permitting display of the test stimulus and matrix simultaneously.

The stimulus cards were of uniform brightness varying in 16 achromatic steps from white to black. A line varying in 16 length steps and/or 16 orientation steps was centered on each card with 1/8-in. (3.18-rmm) red charting tape.

There were seven basic stimulus conditions:

(1) Length (L). The sequence of 16 cards, clockwise or counterclockwise from $L_{1}$ to $L_{16}$ in roughly jnd. units of length (determined on the basis of pilot psychophysical testing), the backgrounds being all of the same brightness and the lines the same orientation (horizontal).

(2) Orientation $(O)$ was varied as the angle of the line with the vertical axis of the stimulus card. There were 16 roughly equal angle steps from the vertical to the horizontal. The length of the lines and the brightness of the background were fixed at the midpoint values of those dimensions.

(3) Brightness (B) varied as 16 shades of Color-aid gray with lines of constant length and orientation.

(4) Length and Orientation, redundant two-dimension. There were four possible matrix arrangements. The two series, number $L_{1}, L_{2} \ldots, L_{16}$ and $O_{1}, O_{2}, \ldots, O_{16}$ were covaried either as $\mathrm{L}_{1} \mathrm{O}_{1}, \mathrm{~L}_{2} \mathrm{O}_{2}, \ldots, \mathrm{L}_{16} \mathrm{O}_{16}$ or as $\mathrm{L}_{1} \mathrm{O}_{16}, \mathrm{~L}_{2} \mathrm{O}_{15}, \ldots, \mathrm{L}_{16} \mathrm{O}_{1}$, and each of these could be ordered either clockwise or counterclockwise on the display board.

(5) Brightness-Orientation redundant two-dimension; also four possible series.

(6) Length-Brightness redundant two-dimension, four sets.

(7) Length-Orientation-Brightness, eight possible arrangements: $\mathrm{L}_{1} \mathrm{O}_{1} \mathrm{~B}_{1}, \ldots, \mathrm{L}_{16} \mathrm{O}_{16} \mathrm{~B}_{16} ; \mathrm{L}_{1} \mathrm{O}_{2} \mathrm{~B}_{16}, \ldots, \mathrm{L}_{16} \mathrm{O}_{36} \mathrm{~B}_{1} ; \mathrm{L}_{1} \mathrm{O}_{16} \mathrm{~B}_{1}$, $\ldots, \mathrm{L}_{10} \mathrm{O}_{1} \mathrm{~B}_{10} ; \mathrm{L}_{16} \mathrm{O}_{1} \mathrm{~B}_{1}, \ldots, \mathrm{L}_{1} \mathrm{O}_{16} \mathrm{~B}_{16}$; each in a clockwise or counterclockwise order.

The particular arrangement $(1,2, \ldots 16$, or $16,15, \ldots 1)$ of any given dimension was balanced across the eight subjects, but for each subject was the same in $1 D, 2 D$, and $3 D$ and under all matrix conditions.

There were four comparative judgment conditions:

(1) Identification (no matrix): The matrix display was present for 3 min of study at the start of the session but was then covered for the remainder of the period. One test stimulus was presented at the center of the board on each trial. Memory demands should have been high in this condition, since the matrix of all possible alternatives appeared only once, at the beginning of the experimental session.

(2) Matrix first: At each trial onset, the subject was presented with the matrix display of 16 stimulus cards mounted (in sequence) on the matrix board. After about $3 \mathrm{sec}$, the cards were covered by moving the mask in front of the board, and at the same instant the center stimulus card was uncovered. Responses were indicated by naming the corresponding matrix card (now covered).

In this condition, memorial demands should have been somewhat lessened, since the display matrix was presented for a brief interval prior to each identification trial. The subject had to make an identification of the stimulus based upon his memory of the total set, but this memory was repeatedly refreshed by presentation of all possible alternatives just before each identification response.

(3) Match-to-sample (matrix last): At each trial onset, the subject was presented with the center stimulus card, the matrix cards being covered by the mask. After about $3 \mathrm{sec}$, the mask was moved, leaving the center card covered and the entire set of 16 matrix cards uncovered.

Here, memory requirements should have been lighter still, since the subject needed only to recall the single test stimulus and compare it to all the possible alternatives which were before him at the time of response.

(4) Discrimination (simultaneous): At each trial onset, the center test stimulus and the set of matrix cards were simul taneously exposed and presented for about $3 \mathrm{sec}$ and then covered.

Here the memory demands were clearly the lightest, since $t\}:$ test stimulus and all the alternatives were physically present at the same time and the subject needed only to visually scan them all to perform the matching task. The task is analogous to a conventional multiple coice discrimination paradigm.

To get the simplest form of the paradigms, the matrix first and match-to-sample delays were set at $0 \mathrm{sec}$, that is, the termination of one and the onset of the other were as simultaneous as our procedures would allow.

Table 1 provides a summary of the experimental conditions and the stimulus conditions for each subject.

\section{Procedure}

The subjects were told that there were two identical sets of cards, one being set up in a matrix (showing matrix board) with the cards numbered 1-16 corresponding to the data sheets given. The data sheets contained blank numbered (1-16) rectangles arranged in a circle, and a set corresponding to the number of trials for each session was available. The subjects were then instructed that the other set of cards would be presented one at a time in the center of the matrix display board and were asked to indicate as rapidly as possible, on the data sheet and orally, the matrix card to which the center card corresponded.

After giving the subjects eight practice trials (using the simultaneous matrix condition), the experimenter explained which matrix condition would be used. A correction procedure was used, the experimenter giving the correct response or saying "correct" immediately. The matrix or stimulus card (depending on the matrix condition) was left exposed until after the correction was given.

There were 80 trials in each experimental condition. The 16 cards were presented in random order five times each, with the restriction that the same card was not presented more than two times in succession.

\section{RESULTS AND DISCUSSION}

The basic analyses were performed on transmitted information scores $(T)$ calculated for each subject in each of the 28 (4 comparative judgment $\times 7$ stimulus) conditions.

Transmitted information is a reliable performance indicator used in our previous studies and one which has proven particularly useful in investigation of dimensionality effects (Fitts \& Posner, 1967; Kaufman $\&$ Levy, 1971). The same analyses were also performed on the response measure of percent correct. The outcome of both sets of analyses were highly comparable (see Table 2), and therefore the results are reported in terms of $T$ only.

Figure 1 illustrates relative performance among the comparative judgment conditions. Three orthogonal comparisons among the four conditions produced the following results: (1) Discrimination (simultaneous) vs. match-to-sample (matrix last), $F(1,189)=4.91, p<.05$. (2) Discrimination and match-to-sample vs. identification (no matrix) and matrix first, $F(1,189)=10.89, p<.001$. (3) Identification vs. matrix first, $F(1,189)<1$.

In general, these results tend to support the notion of a comparative judgment dimension whose endpoints are identification and discrimination. The 
Table 1

Experimental Conditions

Matrix Condition
I. Identification (no matrix)
II. Matrix first with 0 delay
III. Match-to-sample (matrix last with 0 delay)
IV. Discrimination (simultaneous)

Dimensional Condition

1. orientation and brightness constant, vary length $L_{1} \ldots, L_{16}$ or $L_{16} \ldots, L_{1}$

2. brightness and length constant, vary orientation $O_{1} \ldots, O_{16}$ or $O_{16} \ldots, O_{1}$

3. length and orientation constant, vary brightness $B_{1} \ldots, B_{16}$ or $B_{16} \ldots, B_{1}$

4. brightness constant, vary length and orientation $\mathrm{L}_{1} \mathrm{O}_{1}, \mathrm{~L}_{1} \mathrm{O}_{16}, \mathrm{~L}_{16} \mathrm{O}_{1}, \mathrm{~L}_{16} \mathrm{O}_{16}$

5. length constant, vary orientation and brightness $O_{1} B_{1}, O_{1} B_{16}, O_{16} B_{1}, O_{16} B_{16}$

6 . orientation constant, vary brightness and length $B_{1} L_{1}, B_{1} L_{16}, B_{16} L_{1}, B_{16} L_{1}$ 。

7. vary length, orientation, and brightness $L_{1} O_{1} B_{1}, L_{1} O_{1} B_{16}, L_{1} O_{1}{ }_{6} B_{1}, L_{1}{ }_{6} O_{1} B_{1}$, $\mathrm{L}_{1} \mathrm{O}_{16} \mathrm{~B}_{16}, \mathrm{~L}_{16} \mathrm{O}_{16} \mathrm{~B}_{1}, \mathrm{~L}_{16} \mathrm{O}_{1} \mathrm{~B}_{16}, \mathrm{~L}_{16} \mathrm{O}_{16} \mathrm{~B}_{16}$

Dimensional Conditions for Fach Subject

$\begin{aligned} \text { Subject 1: } & \mathrm{L}_{1}, \mathrm{O}_{1}, \mathrm{~B}_{1} \\ & \mathrm{~L}_{1} \mathrm{O}_{1}, \mathrm{O}_{1} \mathrm{~B}_{1}, \mathrm{~B}_{1} \mathrm{~L}_{1} \\ & \mathrm{~L}_{1} \mathrm{O}_{1} \mathrm{~B}_{1}\end{aligned}$

Subject 2: $\mathrm{L}_{1}, \mathrm{O}_{1}, \mathrm{~B}_{16}$

$\mathrm{L}_{1} \mathrm{O}_{1}, \mathrm{O}_{1} \mathrm{~B}_{16}, \mathrm{~B}_{16} \mathrm{~L}$

Subject 3: $\mathrm{L}_{1}, \mathrm{O}_{16}, \mathrm{~B}_{1}$

$\mathrm{L}_{1} \mathrm{O}_{16}, \mathrm{O}_{16} \mathrm{~B}_{1}, \mathrm{~B}_{1} \mathrm{~L}_{1}$

$\mathrm{L}_{1} \mathrm{O}_{16} \mathrm{~B}_{1}$

Subject 4: $L_{1}, O_{16}, B_{1}$ 。

$\mathrm{L}_{1} \mathrm{O}_{16}, \mathrm{O}_{16} \mathrm{~B}_{16}, \mathrm{~B}_{16} \mathrm{~L}$ $\mathrm{L}_{1} \mathrm{O}_{16} \mathrm{~B}_{16}$ $\begin{aligned} \text { Subject 5: } & \mathrm{L}_{16}, \mathrm{O}_{1}, \mathrm{~B}_{1} \\ & \mathrm{~L}_{16} \mathrm{~B}_{1}, \mathrm{O}_{1} \mathrm{~B}_{1}, \mathrm{~B}_{1} \mathrm{~L}_{16} \\ & \mathrm{~L}_{16} \mathrm{O}_{1} \mathrm{~B}_{1}\end{aligned}$

Subject 6: $\mathrm{L}_{16}, \mathrm{O}_{16}, \mathrm{~B}_{1}$ $\mathrm{L}_{16} \mathrm{O}_{16}, \mathrm{O}_{1}{ }_{6} \mathrm{~B}_{1}, \mathrm{~B}_{1} \mathrm{~L}_{1}$ 。

Subject 7: $\mathrm{L}_{16}, \mathrm{O}_{1}, \mathrm{C}_{16}$ $\mathrm{L}_{1}{ }_{6} \mathrm{O}_{1}, \mathrm{O}_{1} \mathrm{~B}_{1 \mathrm{n}}, \mathrm{B}_{16} \mathrm{~L}_{1}$ 。 $\mathrm{L}_{1 \rightarrow} \mathrm{O}_{1} \mathrm{~B}_{16}$

Subject 8: $\mathrm{L}_{16}, \mathrm{O}_{16}, \mathrm{~B}_{16}$ $\mathrm{L}_{16} \mathrm{O}_{16}, \mathrm{O}_{16} \mathrm{~B}_{16}$ $B_{16} L_{16}, L_{16} O_{16} B_{16}$ order of the comparative judgment conditions fell as would have been expected on the basis of consideration of the four paradigms in terms of their memory demands when viewed as the comparison of the test stimulus with an internal representation or schema.

The lowest level of performance is found in the identification task in which the single test stimulus must be compared to the memorial schema of the entire set of stimuli seen as a whole only at the beginning of the experimental session. The refreshing or reconstruction of this schema can only occur on a piecemeal basis, when a test stimulus is presented once on each trial. In the matrix-first condition, the memory demand is still high, since the test stimulus must be compared to a set of stimuli which is only to be found in memory. However, here the entire set is presented immediately prior to the point at which the judgment must be made and memorial reconstruction of the schema might be expected to be somewhat easier. In the match-to-sample paradigm, the demand upon memory is decreased. Only the representation of the single stimulus must be stored, in order to make the comparison between the entire set which is physically present and the test stimulus which is not. The highest level of performance is found in the discrimination condition where the test stimulus and matrix are both physically present and the demand for information storage is light.

The only unusual feature of the data with respect to the preceding analysis is the small and statistically unreliable advantage of the matrix-first condition over identification. If no difference truly exists, the form of the internal representation or the total stimulus set must be fixed early in the experimental session and is resistant to change through repeated presentation of that set. Such stability of judgment standards is inconsistent with the general finding of adaptation level research (Helson, 1964).

Table 2

Average Amounts of Information Transmitted and Percent Correct Responses for Matrix Conditions and Number of Dimensions

\begin{tabular}{|c|c|c|c|c|c|c|c|c|}
\hline \multirow[b]{3}{*}{ Dimensions } & \multicolumn{8}{|c|}{ Matrix Conditions } \\
\hline & \multicolumn{2}{|c|}{ Identification } & \multicolumn{2}{|c|}{ Matrix First } & \multicolumn{2}{|c|}{ Match-to-Sample } & \multicolumn{2}{|c|}{ Discrimination } \\
\hline & $\mathrm{T}$ & $\begin{array}{l}\text { Percent } \\
\text { Correct }\end{array}$ & $\mathrm{T}$ & $\begin{array}{l}\text { Percent } \\
\text { Correct } \\
\end{array}$ & $\mathrm{T}$ & $\begin{array}{l}\text { Percent } \\
\text { Correct } \\
\end{array}$ & $\mathrm{T}$ & $\begin{array}{l}\text { Percent } \\
\text { Correct }\end{array}$ \\
\hline 1 & 2.85 & 55 & 2.90 & 59 & 2.97 & 61 & 3.20 & 71 \\
\hline 2 & 3.03 & 63 & 3.02 & 61 & 3.16 & 68 & 3.45 & 81 \\
\hline 3 & 3.10 & 68 & 3.13 & 70 & 3.41 & 77 & 3.60 & 87 \\
\hline
\end{tabular}



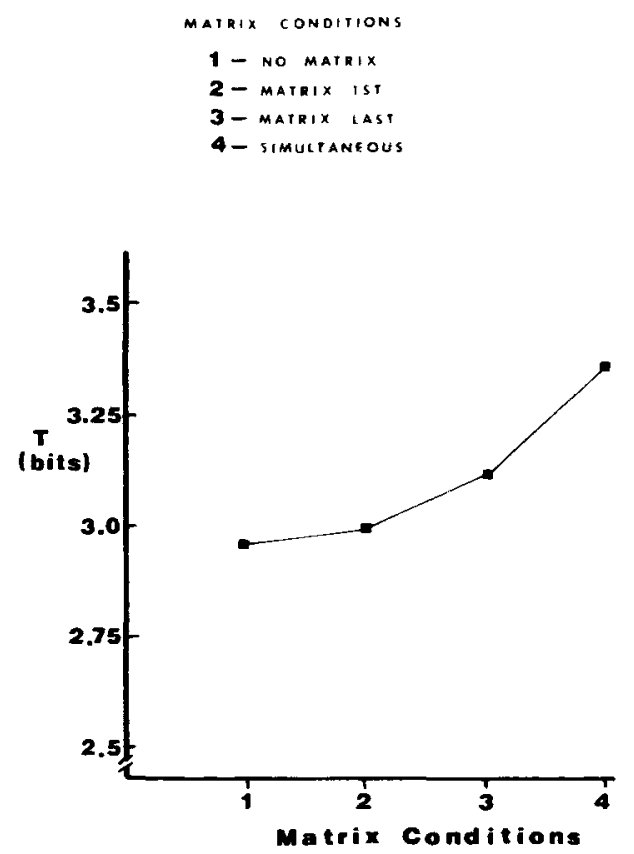

Figure 1. Transmitted information as a function of the matrix condition.

\section{Stimulus Conditions}

The effects of dimensional differences and their relations to judgment conditions are illustrated in Figure 2. The importance of redundancy, often demonstrated in identification and discrimination tasks is again revealed in these two paradigms as well as in the match-to-sample and matrix first paradigm. Over all comparative judgment conditions, threedimensional redundant performance was superior to one or two dimensions, $\mathrm{F}(1,189)=19.92, \mathrm{p}<.001$, and the one-dimensional condition was worse than the two- and three-dimensional cases, $F(1,189)=$ $72.27, \mathrm{p}<.001$.

Within the one-dimensional condition, information transmission for length was much better than for orientation or brightness, $F(1,189)=10.51$, $\mathrm{p}<.005$. This is probably not a result of much intrinsic interest, since it may not reflect so much the qualities of the dimension in general as the limitation of the particular stimulus material. Consistent with the preceding results is that within the twodimensional combinations the only possible difference was that the combination not involving length (orientation and brightness combined) was marginally lower, $F(1,89)=3.35, \mathrm{p}<.10$, than the two combinations involving length.

Consistent with the notion of a single comparative judgment process underlying all four of the experimental tasks studied is the fact that there existed no significant interaction of judgment (matrix) conditions with any of the stimulus (dimensional) condi- tions. The only hint of an interaction can be seen by examination of Figure 2. The slopes of the lower two matrix conditions may be somewhat less steep between $2 \mathrm{D}$ and $3 \mathrm{D}$ than the slopes of the two best matrix conditions. The comparison of this possible difference resulted in an $F$ of $3.35, p<.10$. The results, if replicable, would be noteworthy, since increasing redundancy effects are greater in better matrix conditions (where ceiling effects might be expected to cause a smaller increase) than in lower ones.

The original impetus for the experiment described was our feeling that it was reasonable to study the identifiability of stimuli in a match-to-sample paradigm. Despite the fact that both identification and match-to-sample fall within the conceptual category of complete identification experiments as defined by Bush, Galanter, and Luce (1963), some empirical justification seemed warranted. We feel that such justification is provided by the data of our experiment. While levels of performance between these two paradigms differ, these differences can be accounted for in terms of memorial demands made by the two tasks rather than a process difference. The fact that dimensional effects (number and type of dimensional combinations) are ordered the same way in both conditions speaks to this point.

Performance in the paradigms we have studied order themselves as might be expected in consideration of the test stimulus as part of a comparative judgment with a memorial schema. Increasing memorial demands produce decreasing performance, but more importantly no interactions with di-
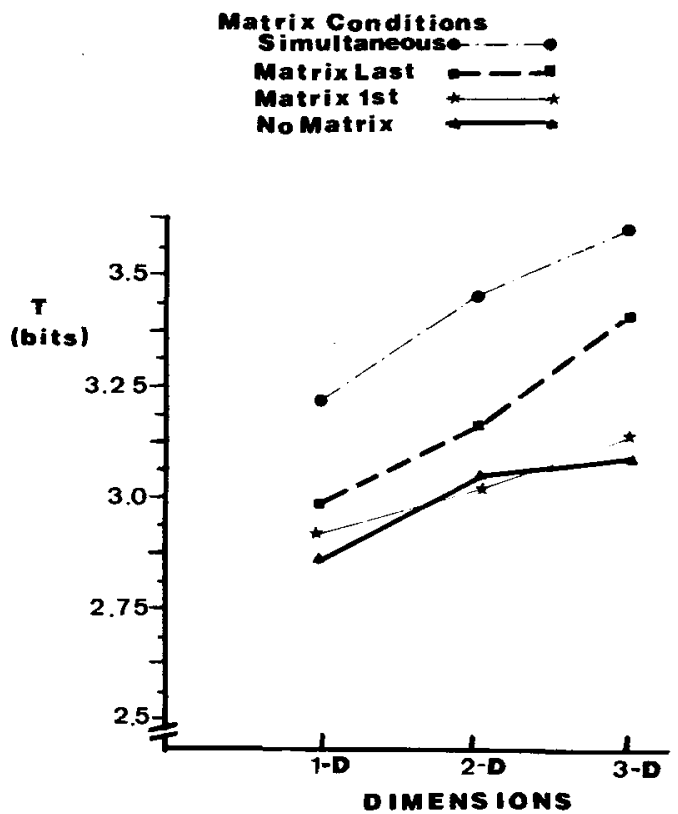

Figure 2. The effects of dimensionality on transmitted information, as a function of matrix condition. 
mensional variation are found. The distinctiveness or identifiability of the stimuli in question would seem to be legitimately investigated under any of the paradigms.

We agree with Leshowitz and Green (1974), who, when taking exception to Siegel and Siegel's (1972) demonstration of the equivalence of paired-associate and absolute judgment paradigms, pointed out that such equivalences do not necessarily solve fundamental questions such as those relating to the limitations on absolute judgment. We disagree, however, with their implication that pointing to such equivalences adds little to our knowledge. Viewing matchto-sample, discrimination and identification as part of a continuum of comparative judgment permits not only a broader view of perceptual phenomena, but also provides increased flexibility in experimentation designed to deal with the distinctiveness of stimuli.

\section{REFERENCES}

Bush, R. R.. Galanter. E., \& Luce, R. D. Characterization and classification of choice experiments. In R. D. Luce, R. R. Bush,
\& E. Galanter (Eds.), Handbook of mathematical psychology (Vol. 1). New York: Wiley, 1963.

FitTs. D. M.. \& Posner. M. I. Human performance. Belmont, Calif: Brooks/Cole. 1967.

Helson, H. Adaptation-level theory. New York: Harper, 1964.

Kalfman. H., \& LeVY, R. M. Analysis of the combination of perceptual dimensions. Psychological Bulletin, 1971, 75, 369-378.

Leshowitz. B., \& Green, D. M. Comments on "Absolute judgment and paired-associate learning: Kissing cousins or identical twins?" Psychological Review, 1974, 81, 177-179.

Miller, G. A. Psychology: The science of mental life. New York: Harper and Row, 1962.

Siegel. J. A.. \& Siegel. W. Absolute judgment and pairedassociate learning: Kissing cousins or identical twins? Psychological Review. 1972, 79. 300-316.

Smith, J.. \& KaUfman, H. Identification capacities of retarded and normal children. Psychonomic Science, 1972, 28, 321-325.

Smith. J., Kaufman. H., Dutch, S., \& Frost, R. Identification by retarded and non-retarded children of stimuli varying along one. two or three dimensions. American Journal of Mental Deficiency, 1975, 80, 191-196.
(Received for publication June 18, 1976; revision accepted December 29, 1976.) 